\title{
Anisotropic Born scattering for qP scalar wavefield using low-rank symbol approximation
}

\author{
lury Araújo ${ }^{1}$, Murillo Nascimento ${ }^{1,2}$, Jessé Costa ${ }^{1,2}$; Alan Souza ${ }^{3}$, and Joerg Schleicher ${ }^{4}$ \\ ${ }^{1}$ Federal University of Pará, UFPA; ${ }^{2}$ National Institute of Petroleum Geophysics, INCT-GP; ${ }^{3}$ PETROBRAS; ${ }^{4}$ University of \\ Campinas, UNICAMP.
}

Copyright 2021, SBGf - Sociedade Brasileira de Geofísica

This paper was prepared for presentation during the $17^{\text {th }}$ International Congress of the Geophysical Society held in Rio de Janeiro, Brazil, 16 to 19 August 2021.

Contents of this paper were reviewed by the Technical Committee of the $17^{\text {th }}$ International Congress of the Brazilian Geophysical Societyldeas and concepts of the text are authors' responsibility and do not necessarily represent any position of the SBGf, its officers or members. Electronic reproduction or storage of any part of this paper for commercial purposes without the written consent of the Brazilian Geophysical Society is prohibited.

\section{Abstract}

We propose Born-scattering operators for modeling qP Born wavefield under pseudo-acoustic approximation for arbitrary anisotropic models. Low-rank approximations are derived for the Bornscattering operators and applied in conjunction with corresponding low-rank evolution operator for modeling the Born scattered wavefield. This procedure avoids pseudo-S artifacts and provides a unified approach for linearizing anisotropic pseudoacoustic evolution operators. Moreover, the scattering operators yield the sensitivity kernels for Born scattering, enabling us to perform the sensitivity analysis of the scattered wavefield relative to anisotropic medium parameters. We validate our implementation in an anisotropic medium with weak contrast among the material properties. Next, we evaluate the Born scattering response for the BP TTI model, the results indicate that our procedure is applicable to very heterogeneous anisotropic media. Finally, the method can be immediately applied to formulate the least-squares migration in pseudo-acoustic anisotropic media.

\section{Introduction}

Seismic imaging techniques require algorithms capable of extrapolating wavefields in time, which is generally done via finite-difference approximations for the acoustic wave equation in isotropic media. Imaging techniques for elastic anisotropic models require the solution of the complete elastic anisotropic wave equation. Although feasible, this approach still has to cope with: crosstalk among wave modes, estimation of a density and a shear-wave model, and a much higher computational and storage cost. In this scenario, pseudo-acoustic approximations for the qP wave mode provides a compromise for imaging in the presence of anisotropy and has been applied extensively by industry (Fletcher et al., 2008).

The $\mathrm{qP}$ wave dispersion relation, under pseudo-acoustic approximation, for VTI media derived by (Alkhalifah, 2000) leads to an fourth-order PDE for the qP wavefield. Its solution honors the kinematic of the corresponding elastic
qP wavefield. Several works then followed, deriving pseudo-acoustic field equations for different classes of anisotropy, as in Fletcher et al. (2008) and Song and Alkhalifah (2012), or different formulations for the pseudoacoustic approximations, as in Du et al. (2008); Zhan et al. (2012); Schleicher and Costa (2016). In general, the pseudo-acoustic approximations can be formulated as a system of two second-order PDEs which respect the $\mathrm{qP}$ wave dispersion relation. As the kinematic properties of the wavefield are preserved in the pseudo-acoustic approximations, these equations are adequate for reverse time migration (Du et al., 2010)) and traveltime tomography (Wang and Sava, 2015).

Pseudo-acoustic equations, unfortunately, generate spurious mode conversion wherever the $\mathrm{qP}$ wavefield is scattered by interfaces or localized heterogeneities (Schleicher and Costa, 2016). The crosstalk between these artifacts and the $\mathrm{qP}$ wavefield can degrade the quality of RTM images. Although artifact-free pseudoacoustic wave equations are available for VTI and TTI media (Schleicher and Costa, 2016), they are not easily extended for general anisotropy. Fomel et al. (2013), using the low-rank approximation theory (Song, 2001), developed a method for extrapolating the pseudo-acoustic wavefield, for arbitrary anisotropic symmetry classes. The low-rank evolution operator matrix, represented in the mixed space-wavenumber domain, leads to a trade off between numerical accuracy, computational, and storage cost (Fomel et al., 2013).

A desirable extension of pseudo-acoustic imaging is the least-squares migration in anisotropic media. Indispensable to achieve this goal is a stable implementation of Born scattering modeling, free from spurious mode conversions. We present a lowrank implementation to compute Born scattering under the pseudo-acoustic approximation absent of mode conversion artifacts. The formulation is general for arbitrary anisotropy classes. The expressions derived for the Born scattering operator contain the sensitivity kernels for each one of medium parameters, allowing us to evaluate their offset dependent contributions to the Born scattered data. Numerical experiments illustrate the decomposition of the scattered wavefield computed with the low-rank approximation of the sensitivity kernels. We use the BP anisotropic salt model to evaluate the method in a strongly heterogeneous model. Computationally, the low-rank Born modeling requires two applications of the evolution operator for each time step and an additional scattering operator for each parameter considered. These scattering operators can be evaluated in parallel. In case 
of a single parameter perturbation the computational and storage cost is about twice the cost for propagating the background wavefield.

\section{Methodology}

In order to avoid mode conversion in the pseudo-acoustic propagation, which plagues the finite-difference solutions of pseudo-acoustic wave equations in anisotropic media, Fomel et al. (2013) proposed an evolution operator of the form

$$
P(\mathbf{x}, t+\Delta t)=\int \hat{P}(\mathbf{k}, t) e^{i \phi(\mathbf{x}, \mathbf{k}, \Delta t)} d \mathbf{k},
$$

where, $P$ is the pseudo-acoustic wavefield, $\mathbf{x}$ the position, $t$ is the time, $\Delta t$ the evolution time interval, $\mathbf{k}$ the wavenumber, and $\phi(\mathbf{x}, \mathbf{k}, \Delta t)$ the phase of the evolution operator specified in the mixed space-wavenumber domain. To first order in $\Delta t, \phi(\mathbf{x}, \mathbf{k}, \Delta t)=\mathbf{k} \cdot \mathbf{x}+|\mathbf{k}| V(\mathbf{x}, \mathbf{k}) \Delta t$, where $V(\mathbf{x}, \mathbf{k})$ is the qP phase velocity (Fomel et al., 2013), which can be determined from the dispersion relation for the qP waves in elastic media or its pseudo-acoustic approximation, i.e.,

$$
P(\mathbf{x}, t+\Delta t)=\int \hat{P}(\mathbf{k}, t) e^{i|\mathbf{k}| V(\mathbf{x}, \mathbf{k}) \Delta t} e^{i \mathbf{k} \cdot \mathbf{x}} d \mathbf{k}+O\left(\Delta^{2} t\right)
$$

To reduce the computation cost by avoiding complex numbers we rewrite the evolution of the wavefield (Fomel et al., 2013),

$$
\begin{aligned}
& P(\mathbf{x}, t+\Delta t)-2 P(\mathbf{x}, t)+P(\mathbf{x}, t-\Delta t)= \\
& 2 \int \hat{P}(\mathbf{k}, t)\left[\cos \left(|\mathbf{k}| V_{0} \Delta t\right)-1\right] e^{i \mathbf{k} \cdot \mathbf{x}} d \mathbf{k}+O\left(\Delta^{2} t\right)
\end{aligned}
$$

\section{Low-rank Born scattering}

We assume the anisotropic medium can be described by $N_{p}$ medium parameters, $\eta_{i}$, with $i \in\left\{1, \ldots, N_{p}\right\}$. Moreover, the medium properties can be decomposed in the form $\eta_{i} \equiv$ $\eta_{i}^{0}+\delta \eta_{i}$, where $\eta_{i}^{0}$ defines a smooth background medium and $\delta \eta_{i}$ the perturbations responsible for the scattering of the wavefield. Consequently, the phase velocity can be expanded as

$$
V\left(\eta_{i}\right)=V_{0}\left(\eta_{i}^{0}\right)+\delta V\left(\eta_{i}^{0}\right)+O\left(\delta^{2} \eta_{i}\right),
$$

where

$$
\delta V\left(\eta_{i}^{0}\right)=\sum_{i=1}^{N_{p}} \frac{\partial V\left(\eta_{i}^{0}\right)}{\partial \eta_{i}} \delta \eta_{i} .
$$

Likewise, we can expand the wavefield in the form $P(\mathbf{x}, t)=$ $P_{0}(\mathbf{x}, t)+\delta P(\mathbf{x}, t)+O\left(\delta^{2} P\right)$, where $P_{0}(\mathbf{x}, t)$ is the wavefield in the background medium. The first order perturbation of the wavefield relative to medium parameters, $\delta P(\mathbf{x}, t)$, defines the Born wavefield, $P_{S}(\mathbf{x}, t) \equiv \delta P(\mathbf{x}, t)$.

The background wavefield obeys the wave equation in the smooth background, therefore, its evolution can be represented as

$$
\begin{aligned}
P_{0}(\mathbf{x}, t+\Delta t)= & 2 P_{0}(\mathbf{x}, t)-P_{0}(\mathbf{x}, t-\Delta t) \\
& +2 \int \hat{P}_{0}(\mathbf{k}, t)\left[\cos \left(|\mathbf{k}| V_{0} \Delta t\right)-1\right] e^{i \mathbf{k} \cdot \mathbf{x}} d \mathbf{k}
\end{aligned}
$$

Using the Born approximation $P(\mathbf{x}, t)=P_{0}(\mathbf{x}, t)+P_{S}(\mathbf{x}, t)$ in equation, retaining only first order perturbations we derived the following evolution scheme for the Born wavefield

$$
\begin{aligned}
P_{S}(\mathbf{x}, t+\Delta t)= & 2 P_{S}(\mathbf{x}, t)-P_{s}(\mathbf{x}, t-\Delta t) \\
& +2 \int \hat{P}_{S}(\mathbf{k}, t)\left[\cos \left(|\mathbf{k}| V_{0} \Delta t\right)-1\right] e^{i \mathbf{k} \cdot \mathbf{x}} d \mathbf{k} \\
& -2 \Delta t \int \hat{P}_{0}(\mathbf{k}, t)|\mathbf{k}| \delta V \sin \left(k V_{0} \Delta t\right) e^{i \mathbf{k} \cdot \mathbf{x}} d \mathbf{k} .
\end{aligned}
$$

\section{Dispersion relation}

The implementation of the low-rank Born scattering, equations 6 and 7 , depends on the phase velocity in the background medium $V_{0}$ and its first order perturbations $\delta V$. To compute those, we start from the dispersion relation for the wave equation in anisotropic elastic media, which governs the phase velocity for a plane wave propagating in a direction $\mathbf{n}$ :

$$
\left|a_{i j k l} s_{j} s_{l}-\delta_{i k}\right|=0
$$

where $a_{i j k l}$ are the components of the density normalized stiffness tensor and $s_{i} \equiv n_{i} / V$ the slowness vector components. This dispersion relation determines a Hamiltonian function (Cerveny, 2005):

$$
\mathscr{H}(\mathbf{x}, \mathbf{s})=0
$$

From the first order perturbation for the Hamiltonian, we can derive $\delta V$ in terms of the perturbations of medium parameters,

$$
\delta V=\sum_{i=1}^{N_{p}} \frac{V}{\left(\mathbf{s} \cdot \nabla_{s} \mathscr{H}\right)} \frac{\partial \mathscr{H}}{\partial \eta_{i}} \delta \eta_{i} .
$$

Substituting equation 10 in the last term of equation 7 we get

$$
\begin{aligned}
& P_{S}(\mathbf{x}, t+\Delta t)=2 P_{S}(\mathbf{x}, t)-P_{S}(\mathbf{x}, t-\Delta t) \\
& \quad+2 \int \hat{P}_{S}(\mathbf{k}, t)\left[\cos \left(|\mathbf{k}| V_{0} \Delta t\right)-1\right] e^{i \mathbf{k} \cdot \mathbf{x}} d \mathbf{k} \\
& \quad-\sum_{i=1}^{N_{p}} \delta \eta_{i} \int \hat{P}_{0}(\mathbf{k}, t) \frac{2|\mathbf{k}| V_{0} \Delta t}{\left(\mathbf{s} \cdot \nabla_{s} \mathscr{H}\right)} \frac{\partial \mathscr{H}}{\partial \eta_{i}} \sin \left(|\mathbf{k}| V_{0} \Delta t\right) e^{i \mathbf{k} \cdot \mathbf{x}} d \mathbf{k} .
\end{aligned}
$$

This representation makes explicit the linear relationship between the scattered wavefield and the perturbations of the material parameters. Note from equation 11 that the perturbations $\delta \eta_{i}$ excite the scattered field. Appendix $\mathbf{A}$ presents the derivatives of the Hamiltonian relative to the medium parameters, equation 11, for mild anisotropic TTI media (Schoenberg and de Hoop, 2000).

The numerical implementation of the evolution operators 6 and 11 using low-rank approximation is presented in appendix B, equations 21 and 22, respectively.

\section{Results and discussion}

\section{Validation of the Algorithm}

The first numerical example validates our approach for the Born scattering (Fomel et al., 2013). The model is a homogeneous TTI block embedded in a homogeneous background. To enforce the accuracy for the Born approximation all the medium properties have a weak contrast. The Table 1 lists the values of the medium 
parameters: the square of $\mathrm{qP}$ wave velocity along the symmetry axis, $C_{0}$, the Thomsen parameters $\varepsilon$ and $\delta$ (Thomsen, 1986), and the angle $\theta$ defining the orientation of the symmetry axis. For computations we use an alternative parameterization for TTI media such that all material parameters have square velocity units, $C_{\varepsilon} \equiv C_{0}(1+$ $2 \varepsilon)$ and $C_{\delta} \equiv C_{0}(1+2 \delta)$ instead of $\varepsilon$ and $\delta$. Figure 1 presents, for the parameter $C_{0}$, the common spatial distribution for all the medium parameters.

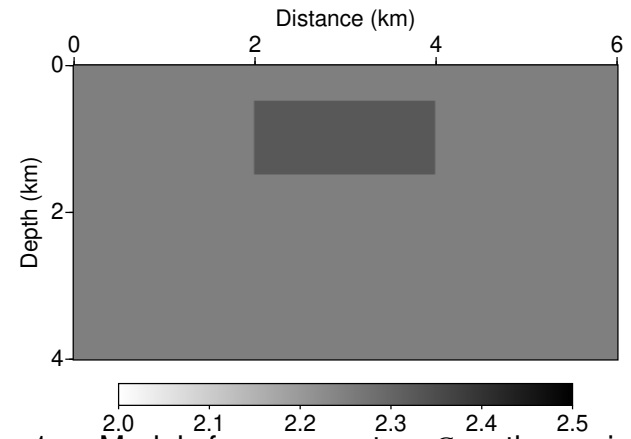

Figura 1: Model for parameter $C_{0}{ }^{2.4}$ the ${ }^{2.5}$ anisotropic parameters $\varepsilon, \delta$ and the orientation of symmetry axis, $\theta$, have the same spatial distribution. Greyscale in $(\mathrm{km} / \mathrm{s})^{2}$.

\begin{tabular}{|c|c|c|}
\hline$\eta_{i}$ & Background & Heterogeneity \\
\hline$C_{0}$ & $2.25(\mathrm{~km} / \mathrm{s})^{2}$ & $2.325(\mathrm{~km} / \mathrm{s})^{2}$ \\
\hline$\varepsilon$ & 0.1 & 0.125 \\
\hline$\delta$ & 0.075 & 0.1 \\
\hline$\theta$ & $27^{\circ}$ & $30^{\circ}$ \\
\hline
\end{tabular}

Tabela 1: TTI medium used for sensitivity analysis.

The low-rank decomposition for this model, with $1 \mathrm{~ms}$ a time step, resulted the same rank for space and wavenumber domains, $N_{x}=N_{k}=4$, for the evolution and scattering operators. To compute the parameters perturbation, $\delta \eta_{i}$, we smoothed each parameter distribution and subtracted it from the corresponding model distribution.

We computed, using the low-rank approximation, a common-shot gather in this model for a source located at $\left(x_{s}=3.0 \mathrm{~km}, z_{s}=0.020 \mathrm{~km}\right)$ and 301 receivers equally spaced of $20 \mathrm{~m}$ at $20 \mathrm{~m}$ depth covering all the model horizontal extension. The source pulse is a Ricker function with peak frequency of $12 \mathrm{~Hz}$. Next, we computed the same common-shot gather using equations 22 and 21 . Figure $2 \mathrm{a}$ presents the exact scattered wavefield, i.e., the difference between the common-shot gather for the complete wavefield $P(\mathbf{x}, t)$ and the background wavefield $P_{0}(\mathbf{x}, t) ; 2 \mathrm{~b}$ shows the scattered Born wavefield $P_{s}(\mathbf{x}, t)$, and Figure $2 c$ contains error of the Born approximation for the scattered field, i.e., the difference between the wavefields in Figures $2 \mathrm{a}$ and $2 \mathrm{~b}$. Figures $2 \mathrm{a}-2 \mathrm{c}$ are plotted in the same scale exemplifying the accuracy of the low-rank Born approximation in weak contrast TTI media.

\section{Sensitivity Analysis}

The low-rank formulation for the Born scattering provides expressions for the sensitivity kernels of each material parameter, second term in the right hand side of equation 11. A nice feature of this formulation is its general and (a)

(b)

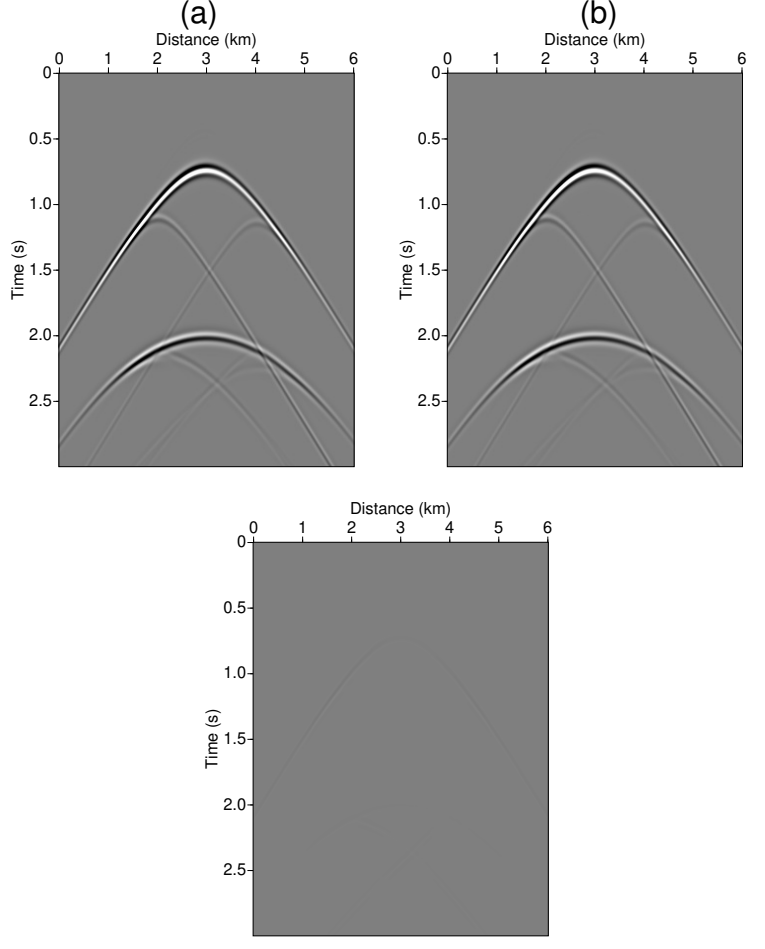

(c)

Figura 2: (a) Exact scattered field, (b) the Born scattered wavefield, (c) Born approximation error.

unified approach for pseudo-acoustic anisotropic medium.

Figure 3 presents the common-shot gathers resulting from each single parameter perturbation. A notable feature of the scattering response of each parameter in Figure $3 a-d$ is the offset dependence variability of the scattering response for each parameter and the lack of symmetry relative to the source position. Moreover, perturbations in the orientation of the symmetry axis produced the smallest contribution to the scattering response. This result illustrates how the sensitivity kernels, determined by the background medium, can assist to improve the conditioning of scattering response linear inversion through a better selection of the parameterization.

\section{Anisotropic BP salt model results}

To evaluate the algorithm in a complex anisotropic model, we computed the scattering response and its Born approximation for a shot gather in the same segment of the BP TTI model presented in Schleicher and Costa (2016). The model spatial distribution for $C_{0}$ is shown in Figure 4.

The low-rank decomposition for the evolution operator, with $1 \mathrm{~ms}$ time step, resulted the same rank for space and wavenumber domains, $N_{x}=N_{k}=7$. An equally accurate low-rank representation for scattering operators required higher ranks, up to 14 for $N_{x}$ and 10 for $N_{k}$. Again, to compute the parameters perturbation, $\delta \eta_{i}$, we smoothed each parameter distribution and subtracted it from the corresponding model distribution.

We computed, using the low-rank approximation, a common-shot gather in this model for a source located at $x_{s}=9.0 \mathrm{~km}$ and $z_{s}=0.020 \mathrm{~km}$. The 901 receivers, equally 
(c)

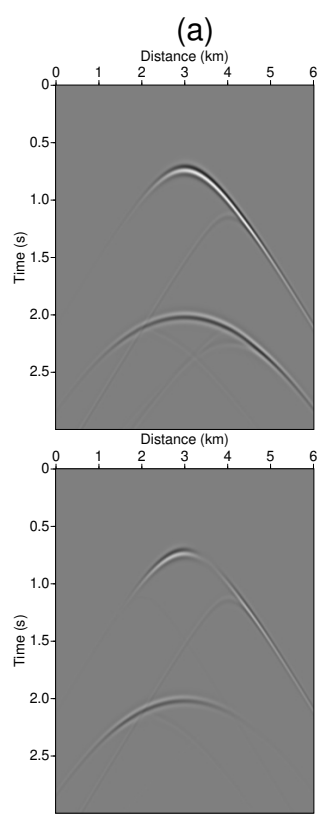

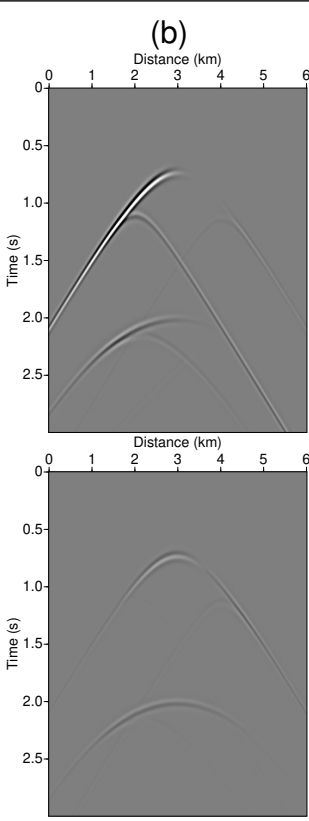

(d)
Figura 3: Contribution of each parameter perturbation to Born scattering: (a) $C_{0}$, (b) $C_{\varepsilon}$, (c) $C_{\delta}$, and (d) $\theta$.

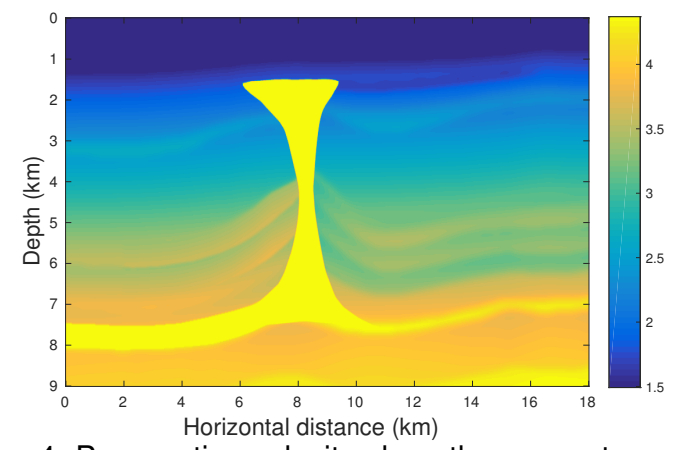

Figura 4: Propagation velocity along the symmetry axis for the segment of the BP salt model used in the numerical experiments.

spaced of $20 \mathrm{~m}$ at $20 \mathrm{~m}$ depth, cover all the model horizontal extension. The source pulse is a Ricker function with peak frequency of $12 \mathrm{~Hz}$. Figure 5a-c contains, respectively, the:(a) scattered wavefield, (b) Born scattered wavefield, (c) the error of Born approximation.

The scattering at the interface between the sediments and the salt body is the dominant event, Figure $5 \mathrm{a}$ and Figure 5b. The large contrast between the medium properties across this interface compromise the accuracy of Born approximation. The Born scattered wavefield for this event, although reproducing accurately the event's exact moveout, does not fit the correct amplitudes. Consequently, Figure $5 \mathrm{c}$ shows that the error of the Born approximation is larger along this event. The error along scattered events produced in the sediments are noticeably smaller. This results indicates that the Born modeling using low-rank decomposition is viable for modeling the scattered seismic wavefield even for a complex anisotropic and heterogeneous models.

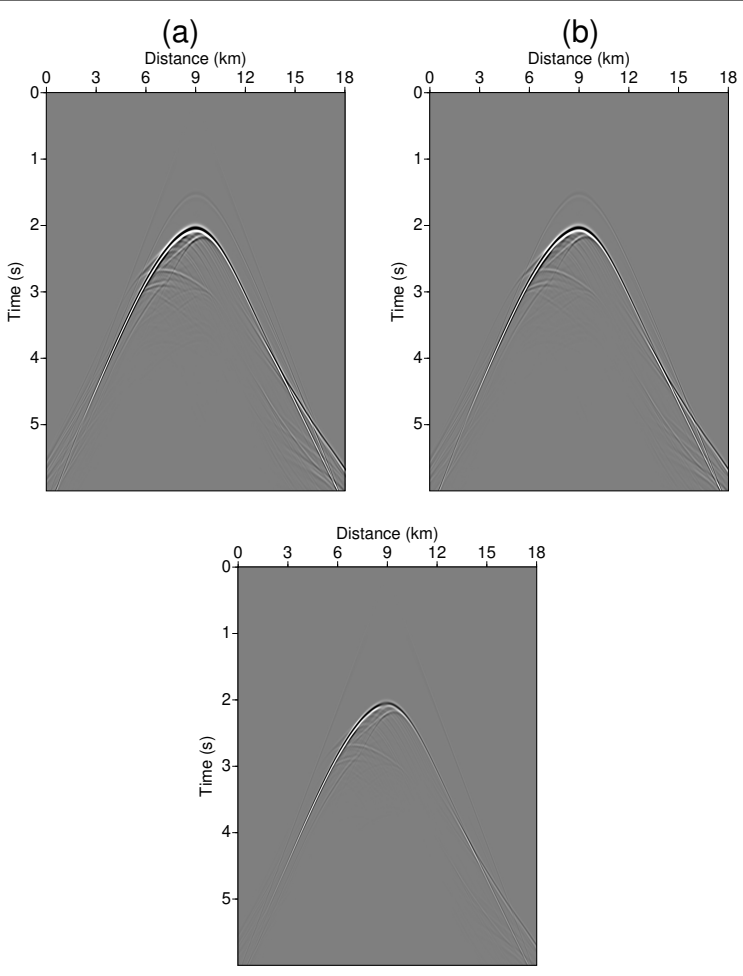

(c)

Figura 5: (a) Exact scattered wavefield, (b) the Born scattered wavefield, and (c) Born approximation error for TTI BP salt model.

\section{Conclusion}

We have implemented Born modeling in anisotropic medium under pseudo-acoustic approximation. Our algorithm uses the low-rank approximation to represent the evolution and wave-scattering operators in the mixed space-wavenumber domain. The formulation presents a general, unified, and straightforward procedure to derive the sensitivity kernels for the Born scattered wavefield. Spurious mode conversions are absent by construction.

The sensitivity analysis presented in the first experiment suggests how the analysis of the sensitivity kernels can help to improve the conditioning of linear inversion of the scattered wavefield, which can be useful for formulating the least-squares migration in TTI media using the pseudoacoustic approximation.

The simulations of Born scattering in the BP salt model highlights the robustness of the proposed method and the dominant role of Born scattering in the reflected wavefield. The computational cost of modeling Born data scales linearly with the number of medium parameters perturbed. If we consider only a single parameter for perturbation, the computational and storage costs are approximately twice the cost for the evolution of the background wavefield. Finally, this approach permits to further explore alternative implementations of least-squares imaging in anisotropic media. 


\section{Acknowledgements}

This work was kindly supported by PETROBRAS. IA, MN and JC also acknowledge the financial support from the Brazilian agencies CNPq and CAPES. We all thank Dr. Cláudio Guerra from PETROBRAS for his incentive and scientific support.

\section{Apendix A: TTI Hamiltonian and its derivatives}

The Hamiltonian we consider for describing the dispersion relation for $\mathrm{qP}$ waves, in mild anisotropic $\mathrm{TI}$ medium (Schoenberg and de Hoop, 2000), is

$$
\begin{aligned}
\mathscr{H}(\mathbf{x}, \mathbf{s}) & =\frac{1}{2}\left[C_{\varepsilon}\left(\mathbf{s} \cdot \mathbf{s}-(\mathbf{s} \cdot \boldsymbol{v})^{2}\right)+C_{0}(\mathbf{s} \cdot \boldsymbol{v})^{2}\right. \\
& \left.-C_{0}\left(C_{\varepsilon}-C_{\delta}\right)\left(\mathbf{s} \cdot \mathbf{s}-(\mathbf{s} \cdot \boldsymbol{v})^{2}\right)(\mathbf{s} \cdot \boldsymbol{v})^{2}-1\right]=0,
\end{aligned}
$$

where $\mathbf{s}$ is the slowness vector, $\boldsymbol{v}$ is the unit vector along the symmetry axis, $C_{0}$ is the square of $\mathrm{qP}$ velocity along the symmetry axis; $C_{\varepsilon}=C_{0}(1+2 \varepsilon)$ and $C_{\delta}=C_{0}(1+2 \delta)$.

The set of parameters chosen to describe the TTI medium are $\eta_{i} \in\left\{C_{0}, C_{\varepsilon}, C_{\delta}, v_{1}\right\}$, so the derivatives of the Hamiltonian with respect to the parameters of the medium can be written as

$$
\begin{aligned}
\frac{\partial \mathscr{H}}{\partial C_{0}} & =\frac{1}{2}(\mathbf{s} \cdot \boldsymbol{v})^{2}\left[1-\left(C_{\varepsilon}-C_{\delta}\right)\left(\mathbf{s} \cdot \mathbf{s}-(\mathbf{s} \cdot \boldsymbol{v})^{2}\right)\right], \\
\frac{\partial \mathscr{H}}{\partial C_{\varepsilon}} & \left.=\frac{1}{2}\left(\mathbf{s} \cdot \mathbf{s}-(\mathbf{s} \cdot \boldsymbol{v})^{2}\right)\left[1-C_{0}(\mathbf{s} \cdot \boldsymbol{v})^{2}\right)\right], \\
\frac{\partial \mathscr{H}}{\partial C_{\delta}} & =\frac{1}{2} C_{0}\left(\mathbf{s} \cdot \mathbf{s}-(\mathbf{s} \cdot \boldsymbol{v})^{2}\right)(\mathbf{s} \cdot \boldsymbol{v})^{2} \text { and } \\
\frac{\partial \mathscr{H}}{\partial v_{1}} & =-\frac{s_{1} v_{3}-s_{3} v_{1}}{v_{3}}(\mathbf{s} \cdot \boldsymbol{v})\left[C_{\varepsilon}-C_{0}\right. \\
& \left.+C_{0}\left(C_{\varepsilon}-C_{\delta}\right)\left(\mathbf{s} \cdot \mathbf{s}-2(\mathbf{s} \cdot \boldsymbol{v})^{2}\right)\right] .
\end{aligned}
$$

Finally,

$$
\mathbf{s} \cdot \nabla_{s} \mathscr{H}=1-C_{0}\left(C_{\varepsilon}-C_{\delta}\right)\left(\mathbf{s} \cdot \mathbf{s}-(\mathbf{s} \cdot \boldsymbol{v})^{2}\right)(\mathbf{s} \cdot \boldsymbol{v})^{2} .
$$

\section{Apendix B: Low-rank symbol approximation}

The numerical low-rank decomposition assumes that an operator $A(\mathbf{x}, \mathbf{k})$, in the mixed space-wavenumber, can be approximated for numerical applications by a matrix $A_{i j} \equiv A\left(\mathbf{x}_{i}, \mathbf{k}_{j}\right)$. For wavefield propagation we must assure the sampling rates in space and wavenumber domains are dense enough to avoid aliasing (Fomel et al., 2013). The low-rank approximation consists in in the factorized representation for $A_{i j}$,

$$
\begin{gathered}
A\left(\mathbf{x}_{i}, \mathbf{k}_{j}\right) \approx \sum_{n=1}^{N_{x}} \sum_{m=1}^{M_{k}} A_{c}\left(\mathbf{x}_{i}, \mathbf{k}_{m}\right) a_{m, n} A_{r}\left(\mathbf{x}_{n}, \mathbf{k}_{j}\right), \\
i \in\{1, \ldots, N\}, j \in\{1, \ldots, M\}
\end{gathered}
$$

where $A_{c}\left(\mathbf{x}_{i}, \mathbf{k}_{n}\right)$ is an orthogonal matrix whose $N_{k}$ columnvectors generate a subspace of the column-space of $A_{i j}$; correspondingly, $A_{r}\left(\mathbf{x}_{m}, \mathbf{k}_{j}\right)$ is an orthogonal matrix whose $N_{x}$ row-vectors generate a subspace of the row-space of $A_{i j}$; the matrix $a_{n m}$ is the low-rank matrix. To be computationally advantageous the low-rank approximation should be accurate for $N_{x}<<N$ and $N_{k}<<M$. In this case, the storage cost is reduced to $N \times M_{k}+M_{k} \times N_{k}+N_{k} \times M$, a much lower cost than the $N \times M$ to store matrix $A_{i j}$. The computational cost to apply the operator $A_{i j}$ is also reduced commensurably.

We applied the algorithm presented in Fomel et al. (2013) to compute the low-rank approximation for the evolution operator (equation 6) and for the scattering operators (equation 11), namely,

$$
E(\mathbf{x}, \mathbf{k}) \equiv 2\left[\cos \left(|\mathbf{k}| V_{0}(\mathbf{x}, \mathbf{k}) \Delta t\right)-1\right]
$$

and

$$
S^{p}(\mathbf{x}, \mathbf{k}) \equiv-\frac{2|\mathbf{k}| V_{0}(\mathbf{x}, \mathbf{k}) \Delta t}{\left(\mathbf{s} \cdot \nabla_{s} \mathscr{H}\right)} \frac{\partial \mathscr{H}}{\partial \eta_{p}} \sin \left(|\mathbf{k}| V_{0}(\mathbf{x}, \mathbf{k}) \Delta t\right)
$$

The low-rank representations of $E(\mathbf{x}, \mathbf{k})$ and $S^{p}(\mathbf{x}, \mathbf{k})$ in leads, respectively, to

$$
\begin{aligned}
& P_{0}\left(\mathbf{x}_{i}, t+\Delta t\right)=2 P_{0}\left(\mathbf{x}_{i}, t\right)-P_{0}\left(\mathbf{x}_{i}, t-\Delta t\right) \\
& \quad+\sum_{m=1}^{M_{k}} E_{c}\left(\mathbf{x}_{i}, \mathbf{k}_{m}\right) \sum_{n=1}^{N_{x}} e_{m n} \sum_{j=1}^{M} E_{r}\left(\mathbf{x}_{n}, \mathbf{k}_{j}\right) \hat{P}_{0}\left(\mathbf{k}_{j}, t\right) e^{i \mathbf{k}_{j} \cdot \mathbf{x}_{i}}
\end{aligned}
$$

and

$$
\begin{aligned}
& P_{S}\left(\mathbf{x}_{i}, t+\Delta t\right)=2 P_{S}\left(\mathbf{x}_{i}, t\right)-P_{S}\left(\mathbf{x}_{i}, t-\Delta t\right) \\
& \quad+\sum_{m=1}^{M_{k}} E_{c}\left(\mathbf{x}_{i}, \mathbf{k}_{m}\right) \sum_{n=1}^{N_{x}} e_{m n} \sum_{j=1}^{M} E_{r}\left(\mathbf{x}_{n}, \mathbf{k}_{j}\right) \hat{P}_{s}\left(\mathbf{k}_{j}, t\right) e^{i \mathbf{k}_{j} \cdot \mathbf{x}_{i}} \\
& \quad+\sum_{p=1}^{N_{p}} \delta \eta_{p} \sum_{m=1}^{M_{k}} S_{c}^{p}\left(\mathbf{x}_{i}, \mathbf{k}_{m}\right) \sum_{n=1}^{N_{x}} s_{m n}^{p} \sum_{j=1}^{M} S_{r}^{p}\left(\mathbf{x}_{n}, \mathbf{k}_{j}\right) \hat{P}_{0}\left(\mathbf{k}_{j}, t\right) e^{i \mathbf{k}_{j} \cdot \mathbf{x}_{i}},
\end{aligned}
$$

where the space and wavenumber domain were sampled in regular meshes. The rank of matrices $A_{c}$ and $A_{r}$ depend on the model heterogeneity and anisotropy.

Application of evolution operator, at each time step, has its computational cost dominated by $N_{x}$ inverse FFTs. Likewise, the application of scattering operators has its computational cost dominated by $N_{x} \times\left(1+N_{p}\right)$ FFTs per time step.

\section{Referências}

Alkhalifah, T., 2000, An acoustic wave equation for anisotropic media: Geophysics, 65, 1239-1250.

Cerveny, V., 2005, Seismic ray theory: Cambridge university press.

Du, X., R. Fletcher, and P. Fowler, 2008, A new pseudo-acoustic wave equation for vti media: 70th EAGE Conference and Exhibition incorporating SPE EUROPEC 2008, European Association of Geoscientists \& Engineers, $\mathrm{cp}-40$.

$\longrightarrow, 2010$, Pure p-wave propagators versus pseudoacoustic propagators for rtm in vti media: 72nd EAGE Conference and Exhibition-Workshops and Fieldtrips, European Association of Geoscientists \& Engineers, $c p-$ 161.

Fletcher, R., X. Du, and P. J. Fowler, 2008, A new pseudo-acoustic wave equation for ti media, in SEG Technical Program Expanded Abstracts 2008: Society of Exploration Geophysicists, 2082-2086.

Fomel, S., L. Ying, and X. Song, 2013, Seismic wave extrapolation using lowrank symbol approximation: Geophysical Prospecting, 61, 526-536. 
Schleicher, J., and J. C. Costa, 2016, A separable strong-anisotropy approximation for pure qp-wave propagation in transversely isotropic mediapure qp-wave propagation: Geophysics, 81, C337-C354.

Schoenberg, M. A., and M. V. de Hoop, 2000, Approximate dispersion relations for qp-qsv-waves in transversely isotropic media: Geophysics, 65, 919-933.

Song, J., 2001, The optimized expression of a high dimensional function/manifold in a lower dimensional space: Chinese Scientific Bulletin, 46, 977-984.

Song, X., and T. Alkhalifah, 2012, A linearized dispersion relation for orthorhombic pseudo-acoustic modeling, in SEG Technical Program Expanded Abstracts 2012: Society of Exploration Geophysicists, 1-6.

Thomsen, L., 1986, Weak elastic anisotropy: Geophysics, 51, 1954-1966.

Wang, H., and P. Sava, 2015, Pseudo-acoustic wavefield tomography with model constraints: CWP Project Review Report, 95-106.

Zhan, G., R. C. Pestana, and P. L. Stoffa, 2012, Decoupled equations for reverse time migration in tilted transversely isotropic media: Geophysics, 77, T37-T45. 